\title{
Neural Network Based Classification of Melanocytic Lesions in Dermoscopy: Role of Input Vector Encoding
}

\author{
Gökhan Ertaş \\ Department of Biomedical Engineering, Yeditepe University, İstanbul, Turkey
}

Cite this article as: G. Ertaş, "Neural Network Based Classification of Melanocytic Lesions in Dermoscopy: Role of Input Vector Encoding”, Electrica, vol. 18, no: 2, pp. 242-248, 2018.

\begin{abstract}
Melanocytic lesions are the main cause of death from skin cancer, and early diagnosis is the key to decreasing the mortality rate. This study assesses the role of input-vector encoding in neural network-based classification of melanocytic lesions in dermoscopy. Twelve dermoscopic measures from 200 melanocytic lesions are encoded by compact encoding, ACD encoding, 1-of-N encoding, normalized encoding, and raw encoding, resulting in five different input-vector sets. Feed-forward neural networks with one hidden layer and one output layer are designed with several neurons in the hidden layer, ranging from two to twenty-two for each type of input-vector set, to classify a melanocytic lesion into common nevus, atypical nevus, and melanoma. Accordingly, 105 networks are designed and trained using supervised learning and then tested by performing a 10 -fold cross validation. All the neural networks achieve high sensitivities, specificities, and accuracies in classification. However, the network with seven neurons in the hidden layer and raw encoded dermoscopic measures as the input vector realizes the highest sensitivity (97.0\%), specificity (98.1\%), and accuracy (98.0\%). The practical use of the network can facilitate lesion classification by retaining the needed expertise and minimizing diagnostic variability among dermatologists.
\end{abstract}

Keywords: Dermoscopy, melanocytic lesion, multilayer perceptron, encoding, classification

\section{Corresponding Author: \\ Gökhan Ertaş}

\section{E-mail:}

gokhan.ertas@yeditepe.edu.tr

Received: 23.02.2018

Accepted: 31.05 .2018

(c) Copyright 2018 by Electrica

Available online at

http://electrica.istanbul.edu.tr

DOI: 10.26650/electrica.2018.97856

\section{Introduction}

Melanocytic lesions are the pigmented lesions of the human skin that require utmost attention due to the risk of skin cancer. During cancer development, the cells of these lesions change their behavior resulting in different types of lesions: common nevus (the most benign lesion), atypical nevus (benign lesion but mimics most of the physical characteristics of malignancy) and melanoma (the malignant lesion). Melanoma is the most aggressive and life-threatening form of skin cancer [1]. To cure melanomas, current drug discoveries and targeted therapies indicate evidence for possible opportunities. However, drug resistance can make the outcomes cumbersome [2]. Early detection and diagnosis still remain the key to decreasing the mortality rate [3].

In diagnosis of melanocytic lesions, especially in detecting the early phase of melanoma, dermoscopy is reported to be a beneficial technique that may reveal the morphological structures and patterns of a lesion by performing imaging based on optical principles [4]. The images captured are evaluated by experienced dermatologists to determine several dermoscopic measures which are next entered to an algorithm for cancer diagnosis. The ABCD rule, the Menzies method and the 7-point checklist are among the main algorithms used [5-7].

The $A B C D$ method relies on the interpretation of the measures of asymmetrical lesion shape, lesion border, number of colors and presence of different structural components in the lesion [5]. A total dermoscopy score (TDS) is calculated using a formula that incorporates points assessed for each measure. Diagnosis is made with respect to community-recognized thresholds. In Menzies scoring, the measures of asymmetry of colors and/or structures in the lesion are considered in adjunct to the number of colors and the presence 
Table 1. Dermoscopic measures and attributed values

\begin{tabular}{|c|c|c|c|c|}
\hline \multicolumn{2}{|l|}{ Measure } & Symbol & Value & Meaning \\
\hline \multicolumn{2}{|l|}{ Asymmetry } & $m_{a}$ & $0 / 1 / 2$ & 0: Symmetric, 1: Symmetric in 1-axes or 2: Asymmetric \\
\hline \multirow[t]{6}{*}{ Color } & white & $m_{c}{ }^{w}$ & $0 / 1$ & 0 : Absent or 1 : Present \\
\hline & red & $m_{c}^{r}$ & $0 / 1$ & $0:$ Absent or 1 : Present \\
\hline & light brown & $m_{c}^{l b}$ & $0 / 1$ & 0 : Absent or 1: Present \\
\hline & dark brown & $m_{c}^{d b}$ & $0 / 1$ & 0 : Absent or 1 : Present \\
\hline & blue-gray & $m_{c}^{b g}$ & $0 / 1$ & 0 : Absent or 1: Present \\
\hline & black & $m_{c}^{b}$ & $0 / 1$ & 0 : Absent or 1 : Present \\
\hline \multirow[t]{5}{*}{ Structure } & pigment network & $m_{p}$ & $0 / 1$ & 0: Atypical or 1:Typical \\
\hline & dots/globules & $m_{d g}$ & $0 / 1 / 2$ & 0: Absent, 1: Atypical or 2: Typical \\
\hline & streaks & $m_{s}$ & $0 / 1$ & $0:$ Absent or 1 : Present \\
\hline & regression areas & $m_{r}$ & $0 / 1$ & $0:$ Absent or 1: Present \\
\hline & blue-whitish veil & $m_{v}$ & $0 / 1$ & 0 : Absent or 1: Present \\
\hline
\end{tabular}

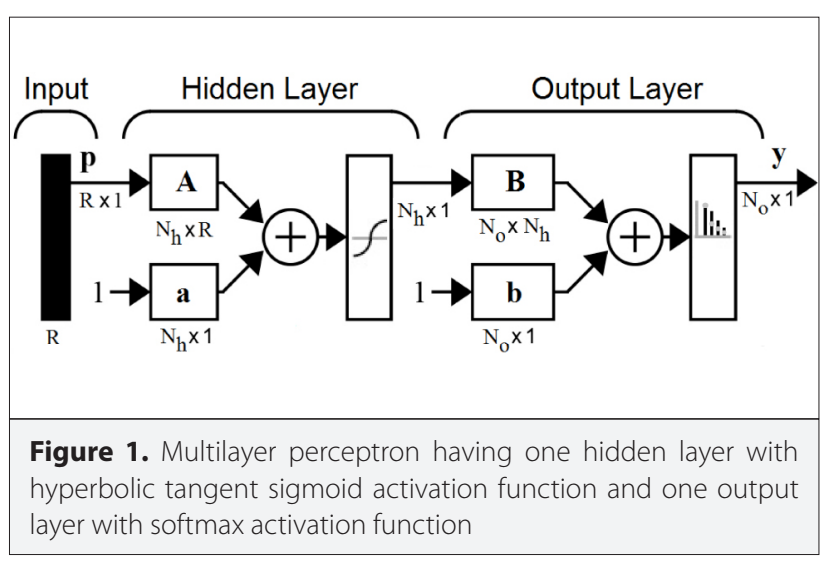

of different structural components in the lesion [6]. All of the measures are scored as categorically present or absent. A decision-tree based approach is used to diagnose malignancy. A 7-point checklist considers the presence of different structural components in the lesion as the measures [7]. Any measure may take one or two points depending on its pre-defined importance. A total score is given by calculating the points taken. Diagnosis is made with respect to a community-recognized threshold value. The performances of the algorithms mentioned above are limited, and recently machine learning based algorithms have been gaining increased interest to improve classification of melanocytic lesions in dermoscopy [8-11].

In this study, we aim to assess the performances of several feed-forward neural networks in classifying melanocytic lesions considering dermoscopic measures encoded by different schemes as the inputs to the networks.

\section{Materials and Methods}

\section{Study Dataset}

This retrospective study employs $\mathrm{PH} 2$ dataset established by a group of researchers from the Technical Universities of Porto and the Dermatology Service of Pedro Hispano Hospital in Portugal to be used as ground truth in the evaluation of classification algorithms [12]. The dataset covers 200 melanocytic lesions: 80 common nevi, 80 atypical nevi and 40 melanomas. For each lesion, a total of 12 measures extracted from the dermoscopic image of the lesion are provided. These measures are listed with attributed values in Table 1.

\section{Neural Network Design}

The current study involves the design of multiple feed-forward neural networks inherited from a multilayer perceptron with one hidden layer and one output layer as seen in Figure 1. The output vector, $\mathbf{y}$ is written in terms of the input vector, $\mathbf{p}$ using

$$
\mathbf{y}=\mathrm{f}(\mathbf{p})=\operatorname{softmax}(\mathbf{B} \tanh (\mathbf{A p}+\mathbf{a})+\mathbf{b})
$$

here $\mathbf{A}$ and $\mathbf{a}$ denote the matrix of weights for the neurons and the bias vector in the hidden layer while $\mathbf{B}$ and $\mathbf{b}$ are the matrix of weights for the neurons and the bias vector in the output layer, respectively. The hidden layer owns a hyperbolic tangent sigmoid activation function while a softmax activation function sits in the output layer. The dimensions of the matrices and the length of the vectors are assigned by the number of elements in the input vector (R), the number of neurons placed in the hidden layer $\left(\mathrm{N}_{h}\right)$, and the number of neurons placed in the output layer $\left(\mathrm{N}_{\mathrm{o}}\right)$. 
In the current study, considering the three different classes of melanocytic lesions, namely common nevus, atypical nevus and melanoma, $\mathrm{N}_{\mathrm{o}}$ was set to three. However, $\mathrm{N}_{\mathrm{h}}$ was set to range between two and twenty-two to test the classification performance with respect to the number of neurons in the hidden layer and $\mathrm{R}$ took values from three to sixteen according to the five different input vector sets studied.

\section{Input Vector Encoding}

The input vector for a neural network was formed by applying four different encoding schemes to the measures supplied in the study dataset for the melanocytic lesions. These are compact encoding, ACD encoding, 1-of- $\mathrm{N}$ encoding, normalized scale encoding and raw encoding. In the raw encoding scheme, all the measures are directly employed in the input vector. The normalized scale encoding is very similar to raw encoding, i.e. all the measures are directly used in the input vector, but they are divided by their possible maximum values so that all the measures are limited to be between 0 and 1. The 1-of- $\mathrm{N}$ encoding considers binary values for any measure and therefore for the measures taking more than two values supplementary measures are defined as:

$$
\begin{aligned}
& \text { if } \mathrm{m}_{\mathrm{a}}=\left\{\begin{array}{llll}
0 ; & \mathrm{m}_{\mathrm{a}, 0}=1 & \mathrm{~m}_{\mathrm{a}, 1}=0 & \mathrm{~m}_{\mathrm{a}, 2}=0 \\
1 ; & \mathrm{m}_{\mathrm{a}, 0}=0 & \mathrm{~m}_{\mathrm{a}, 1}=1 & \mathrm{~m}_{\mathrm{a}, 2}=0 \\
2 ; & \mathrm{m}_{\mathrm{a}, 0}=0 & \mathrm{~m}_{\mathrm{a}, 1}=0 & \mathrm{~m}_{\mathrm{a}, 2}=1
\end{array}\right\} \\
& \text { if } \mathrm{m}_{\mathrm{dg}}=\left\{\begin{array}{llll}
0 ; & \mathrm{m}_{\mathrm{dg}, 0}=1 & \mathrm{~m}_{\mathrm{dg}, 1}=0 & \mathrm{~m}_{\mathrm{dg}, 2}=0 \\
1 ; & \mathrm{m}_{\mathrm{dg}, 0}=0 & \mathrm{~m}_{\mathrm{dg}, 1}=1 & \mathrm{~m}_{\mathrm{dg}, 2}=0 \\
2 ; & \mathrm{m}_{\mathrm{dg}, 0}=0 & \mathrm{~m}_{\mathrm{dg}, 1}=0 & \mathrm{~m}_{\mathrm{dg}, 2}=1
\end{array}\right\}
\end{aligned}
$$

$A C D$ encoding relies on the concept of the well-recognized $A B C D$ evaluation method that looks at the total number of colors among the pre- defined color types and the total number of structures among the pre-defined structure types present within the lesion [5]. To implement this method, supplementary measures are defined as follows:

$$
\begin{aligned}
& m_{C}=m_{c}^{\mathrm{w}}+m_{c}^{r}+m_{c}^{l b}+m_{c}^{d b}+m_{c}^{b g}+m_{c}^{b} \\
& m_{D}=m_{p}+m_{d g}+m_{s}+m_{r a}+m_{v}
\end{aligned}
$$

The compact encoding method compresses the measure data while performing normalization preserving all measures. The following measures obtained from the raw measures are offered by this method:

$$
m_{C C}=\frac{m_{c}^{w}+2 m_{c}^{r}+4 m_{c}^{l b}+8 m_{c}^{d b}+16 m_{c}^{b g}+32 m_{c}^{b}}{63}
$$

$m_{D C}=\frac{m_{p}+2 m_{d g, 1}+4 m_{d g, 2}+8 m_{s}+16 m_{r a}+32 m_{v}}{63}$

Table 2 shows the input vector and the number of elements in the input vector obtained for each encoding scheme studied.

\section{Neural Network Training and Testing}

For each neural network designed, training and testing tasks were performed using 10-fold cross validation [13]. The input vector set covering all cases in the study dataset was first divided into ten partitions. Next, nine of these partitions were assigned to the training dataset while the remaining one partition was allocated as the test dataset.

Using the training dataset, a supervised based training was initiated by assigning weights for the neurons and the bias randomly and the optimal values for the weights and the bias were obtained by a backpropagation algorithm using scaled conjugate gradient optimization and cross-entropy loss function between the outputs of the network and the target out-

\begin{tabular}{|c|c|c|c|c|c|}
\hline Encoding & Raw & Normalized & 1-of-N & $A C D$ & Compact \\
\hline \multirow{16}{*}{$p$} & $\mathrm{~m}_{\mathrm{a}}$ & $\mathrm{m}_{\mathrm{a}} / 2$ & $\mathrm{~m}_{\mathrm{a}^{\prime}} 0$ & $m_{a}$ & $\mathrm{~m}_{\mathrm{a}} / 2$ \\
\hline & $m_{c}^{w}$ & $m_{c}^{w}$ & $m_{a^{\prime}} 1$ & $m_{c}$ & $m_{c c}$ \\
\hline & $m_{c}^{r}$ & $m_{c}^{r}$ & $m_{a^{\prime}} 2$ & $m_{D}$ & $m_{D C}$ \\
\hline & $\mathrm{m}_{\mathrm{c}}^{\mathrm{lb}}$ & $\mathrm{m}_{\mathrm{c}}^{\mathrm{lb}}$ & $m_{c}^{w}$ & & \\
\hline & $m_{c}^{d b}$ & $m_{c}^{d b}$ & $m_{c}^{r}$ & & \\
\hline & $m_{c}^{b g}$ & $\mathrm{~m}_{\mathrm{c}}^{\mathrm{bg}}$ & $\mathrm{m}_{\mathrm{c}}^{\mathrm{lb}}$ & & \\
\hline & $m_{c}^{b}$ & $m_{c}^{b}$ & $m_{c}^{d b}$ & & \\
\hline & $m_{p}$ & $m_{p}$ & $m_{c}^{b g}$ & & \\
\hline & $m_{d g}$ & $m_{d g} / 2$ & $m_{c}^{b}$ & & \\
\hline & $\mathrm{m}_{\mathrm{s}}$ & $\mathrm{m}_{\mathrm{s}}$ & $m_{p}$ & & \\
\hline & $\mathrm{m}_{\mathrm{r}}$ & $\mathrm{m}_{\mathrm{r}}$ & $\mathrm{m}_{\mathrm{dg}, 0}$ & & \\
\hline & $m_{v}$ & $m_{v}$ & $\mathrm{~m}_{\mathrm{dg}, 1}$ & & \\
\hline & & & $\mathrm{m}_{\mathrm{dg}, 2}$ & & \\
\hline & & & $m_{s}$ & & \\
\hline & & & $m_{r}$ & & \\
\hline & & & $m_{v}$ & & \\
\hline $\mathrm{R}$ & 12 & 12 & 16 & 3 & 3 \\
\hline
\end{tabular}
puts (i.e. lesion classes) $[14,15]$. The training was stopped when the number of epochs reached its maximum of $10^{3}$ or the gradient of the cross-entropy reached its minimum of $10^{-10}$. Follow-

Table 2. Encoding schemes and resulting input vectors 
ing training, the network was tested with the testing dataset unknown to the network. The outputs of the network were recorded to assess the classification performance of the network. The folding process, explained in the details above, was repeated ten times to be able to consider each partition generated during the 10 -fold cross validation as the test dataset.

\section{Assessing the Performance of Classification}

Classification performance is assessed by sensitivity (Se), specificity (Sp) and accuracy (Acc) metrics obtained by computing the arithmetic mean of these metrics determined for each folding process. For the $\mathrm{k}$-th folding process, the overall sensitivity, specificity and accuracy for that fold can be calculated using

$$
\begin{aligned}
& \operatorname{Se}(\mathrm{k})=\sum_{\mathrm{n}}(\operatorname{Pr}(\mathrm{n}) \times \operatorname{Se}(\mathrm{k}, \mathrm{n})) \\
& \operatorname{Sp}(\mathrm{k})=\sum_{\mathrm{n}}(\operatorname{Pr}(\mathrm{n}) \times \operatorname{Sp}(\mathrm{k}, \mathrm{n})) \\
& \operatorname{Acc}(\mathrm{k})=\sum_{\mathrm{n}}(\operatorname{Pr}(\mathrm{n}) \times \operatorname{Acc}(\mathrm{k}, \mathrm{n}))
\end{aligned}
$$

Here $\mathrm{n}$ denotes the class number and ranges from 1 to $3 \mathrm{ac}-$ cording to the three classes ( $c_{1}$ : common nevus, $c_{2}$ : atypical nevus and $c_{3}$ : melanoma). $\operatorname{Pr}(n)$ is the probability of a lesion being in class $n$. Se(k,n), Sp $(k, n)$ and $A c c(k, n)$ are calculated considering the classification results by the neural network for the $n$-th class for the k-th fold by [16]

$$
\operatorname{Se}(k, n)=\frac{\text { Number of true-positive detections for } c_{n}}{\text { Number of lesions in } c_{n}} \times 100
$$

$$
\operatorname{Sp}(k, n)=\frac{\text { Number of true-negative detections for } \mathrm{c}_{\mathrm{n}}}{\text { Number of lesions not in } \mathrm{c}_{\mathrm{n}}} \times 100
$$

$$
\operatorname{Acc}(k, n)=\frac{\begin{array}{c}
\text { Number of true-positive }+ \\
\text { true-negative detections for } c_{n}
\end{array}}{\text { Total number of lesions }} \times 100
$$

The neural networks designed were numerically implemented and classifications were performed on a standard desktop PC (Intel i5-4460 3.20GHz processor, 6GB memory and 64-bit operating system) using our in-house computer software tools developed using MATLAB (v8.2; Natick, MA).

\section{Results}

A total of five different input vectors were analyzed in the classification of melanocytic lesions into common nevus, atypical nevus and melanoma by feed-forward neural networks having one hidden layer and one output layer inherited from a multi-layer perceptron. Each type of input vector was fed into a dedicated neural network while the number of neurons in the hidden layer of the network was changed from two to twenty-two. This led to the design and analysis of 105 neural networks in total.

Table 3 tabulates the classification performances of the neural networks fed by the input vectors formed using raw encoding, normalized scale encoding, 1-of-N encoding, ACD encoding and compact encoding with respect to the number of neurons employed in the hidden layer of the networks. Corresponding plots for the performances are seen in Figures $2 a-2 c$. The raw encoding and the normalized scale encoding let the neural networks provide the same very high sensitivities (96.1\%-97.0\%), specificities (97.7\%-98.1\%) and accuracies (97.4\%-98.0\%). On the other hand, the neural networks fed by the input vectors formed using 1 -of-N encoding achieve very high sensitivities (95.9\%-97.1\%), specificities (97.5\%-98.1\%) and accuracies (97.2\%-98.0\%), too. However, ACD encoding induces lower sensitivities (85.9\%-87.5\%), specificities $(91.8 \%$ $92.5 \%$ ) and accuracies (90.3\%-91.3\%). For compact encoding, a wide range of sensitivities, specificities and accuracies are determined, and better values are obtained when the numbers of neurons in the hidden layer of the networks are increased. In

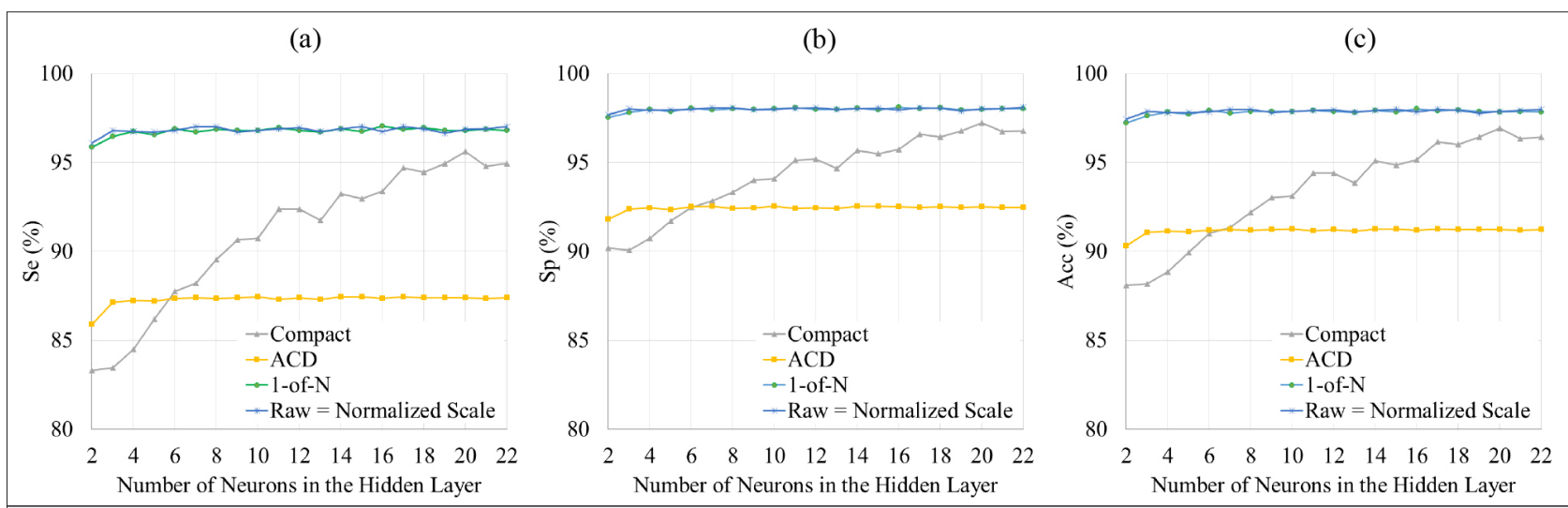

Figure 2. a-c. Plots for classification sensitivity (a); specificity (b) and accuracy obtained for the neural networks (c) 
Table 3. Classification performances of the neural networks for the encoding schemes

\begin{tabular}{|c|c|c|c|c|c|c|c|c|c|c|c|c|c|c|c|}
\hline \multirow[b]{2}{*}{$\mathrm{Nh}$} & \multicolumn{3}{|c|}{ Raw Encoding } & \multicolumn{3}{|c|}{ Normalized Encoding } & \multicolumn{3}{|c|}{ 1-of-N Encoding } & \multicolumn{3}{|c|}{ ACD Encoding } & \multicolumn{3}{|c|}{ Compact Encoding } \\
\hline & $\mathrm{Se}$ & Sp & Acc & Se & Sp & Acc & Se & Sp & Acc & Se & Sp & Acc & Se & Sp & Acc \\
\hline 2 & 96.1 & 97.7 & 97.4 & 96.1 & 97.7 & 97.4 & 95.9 & 97.5 & 97.2 & 85.9 & 91.8 & 90.3 & 83.3 & 90.2 & 88.1 \\
\hline 3 & 96.8 & 98.0 & 97.9 & 96.8 & 98.0 & 97.9 & 96.5 & 97.8 & 97.6 & 87.2 & 92.4 & 91.1 & 83.5 & 90.1 & 88.2 \\
\hline 4 & 96.8 & 97.9 & 97.8 & 96.8 & 97.9 & 97.8 & 96.8 & 98.0 & 97.8 & 87.3 & 92.5 & 91.1 & 84.5 & 90.7 & 88.8 \\
\hline 5 & 96.7 & 98.0 & 97.8 & 96.7 & 98.0 & 97.8 & 96.6 & 97.9 & 97.7 & 87.2 & 92.4 & 91.1 & 86.2 & 91.7 & 89.9 \\
\hline 6 & 96.8 & 98.0 & 97.9 & 96.8 & 98.0 & 97.9 & 96.9 & 98.1 & 97.9 & 87.4 & 92.5 & 91.2 & 87.8 & 92.5 & 91.0 \\
\hline 7 & 97.0 & 98.1 & 98.0 & 97.0 & 98.1 & 98.0 & 96.7 & 97.9 & 97.8 & 87.4 & 92.5 & 91.2 & 88.2 & 92.8 & 91.4 \\
\hline 8 & 97.0 & 98.1 & 98.0 & 97.0 & 98.1 & 98.0 & 96.9 & 98.0 & 97.9 & 87.4 & 92.4 & 91.2 & 89.6 & 93.3 & 92.2 \\
\hline 9 & 96.7 & 97.9 & 97.8 & 96.7 & 97.9 & 97.8 & 96.8 & 98.0 & 97.9 & 87.4 & 92.5 & 91.2 & 90.7 & 94.0 & 93.0 \\
\hline 10 & 96.8 & 98.0 & 97.9 & 96.8 & 98.0 & 97.9 & 96.8 & 98.0 & 97.9 & 87.5 & 92.6 & 91.3 & 90.8 & 94.1 & 93.1 \\
\hline 11 & 96.9 & 98.0 & 97.9 & 96.9 & 98.0 & 97.9 & 97.0 & 98.1 & 97.9 & 87.3 & 92.4 & 91.2 & 92.4 & 95.1 & 94.4 \\
\hline 12 & 97.0 & 98.1 & 98.0 & 97.0 & 98.1 & 98.0 & 96.8 & 98.0 & 97.9 & 87.4 & 92.5 & 91.2 & 92.4 & 95.2 & 94.4 \\
\hline 13 & 96.8 & 98.0 & 97.8 & 96.8 & 98.0 & 97.8 & 96.7 & 98.0 & 97.8 & 87.3 & 92.4 & 91.2 & 91.8 & 94.7 & 93.8 \\
\hline 14 & 96.9 & 98.0 & 97.9 & 96.9 & 98.0 & 97.9 & 96.9 & 98.0 & 97.9 & 87.5 & 92.5 & 91.3 & 93.3 & 95.7 & 95.1 \\
\hline 15 & 97.0 & 98.1 & 98.0 & 97.0 & 98.1 & 98.0 & 96.8 & 98.0 & 97.8 & 87.5 & 92.5 & 91.3 & 93.0 & 95.5 & 94.9 \\
\hline 16 & 96.8 & 98.0 & 97.8 & 96.8 & 98.0 & 97.8 & 97.1 & 98.1 & 98.0 & 87.4 & 92.5 & 91.2 & 93.4 & 95.7 & 95.2 \\
\hline 17 & 97.0 & 98.1 & 98.0 & 97.0 & 98.1 & 98.0 & 96.9 & 98.0 & 97.9 & 87.5 & 92.5 & 91.3 & 94.7 & 96.6 & 96.2 \\
\hline 18 & 96.9 & 98.0 & 97.9 & 96.9 & 98.0 & 97.9 & 97.0 & 98.1 & 98.0 & 87.4 & 92.5 & 91.2 & 94.5 & 96.4 & 96.0 \\
\hline 19 & 96.7 & 97.9 & 97.8 & 96.7 & 97.9 & 97.8 & 96.8 & 98.0 & 97.9 & 87.4 & 92.5 & 91.2 & 95.0 & 96.8 & 96.4 \\
\hline 20 & 96.9 & 98.0 & 97.9 & 96.9 & 98.0 & 97.9 & 96.8 & 98.0 & 97.9 & 87.4 & 92.5 & 91.2 & 95.6 & 97.2 & 96.9 \\
\hline 21 & 96.9 & 98.0 & 97.9 & 96.9 & 98.0 & 97.9 & 96.9 & 98.0 & 97.9 & 87.4 & 92.5 & 91.2 & 94.8 & 96.7 & 96.3 \\
\hline 22 & 96.9 & 98.0 & 97.9 & 96.9 & 98.0 & 97.9 & 96.8 & 98.0 & 97.9 & 87.4 & 92.5 & 91.2 & 95.0 & 96.8 & 96.4 \\
\hline
\end{tabular}

contrast to this, very similar performance values are offered by the networks fed by the input vectors formed using the other encoding schemes, independent of the number of neurons in the hidden layer.

The highest classification accuracy value is considered to determine the best classification performance achieved for each encoding scheme. Raw and the normalized scale encodings offer the same best classification performance: $\mathrm{Se}=97.0 \%, \mathrm{Sp}=$ $98.1 \%$ and $A c c=98.0 \%$. This performance is provided by two networks both employing seven neurons in the hidden layer. The 1-of- $\mathrm{N}$ encoding offers the best performance with $\mathrm{Se}=$ 97.1\%, $\mathrm{Sp}=98.1 \%$ and $\mathrm{Acc}=98.0 \%$ and a network having ten hidden neurons delivers this performance. For the ACD encoding, the best performance is $\mathrm{Se}=87.5 \%, \mathrm{Sp}=92.6 \%$ and $\mathrm{Acc}=$ $91.3 \%$ by a network with ten hidden neurons. The compact encoding delivers the best performance as $\mathrm{Se}=95.6 \%, \mathrm{Sp}=97.2 \%$ and $\mathrm{Acc}=96.9 \%$ when a network having twenty hidden neurons is considered.

\section{Conclusion}

Feed-forward neural networks obtained from a multilayer perceptron with one hidden layer and one output layer can classify melanocytic lesions from dermoscopic measures of the lesions with quite high sensitivity, high specificity and high accuracy. However, the specificity is always higher than the sensitivity. The input vectors of the networks can be formed by applying different encoding schemes to the measures. This process requires utmost attention since it has a remarkable impact on the classification performance.

The ACD encoding that relies on the concept of well-recognized $A B C D$ evaluation method offers limited classification sensitivity, 
specificity and accuracy. This is due to the fact that the $A B C D$ method looks at the dermoscopic measures as the asymmetry of the lesion, the total number of colors among the pre- defined color types and the total number of structures among the pre-defined structure types present within the lesion. When compared to the ACD encoding, the compact encoding reveals better sensitivity, specificity and accuracy. This may be as a result of using normalized lesion asymmetry in addition to the compressed and then normalized data of the presence of each color and each pre-defined structure within the lesion separately. On the other hand, the raw encoding that relies on direct use of the lesion asymmetry and the presence of each color and each pre-defined structure within the lesion as the measures delivers the highest sensitivity, specificity and accuracy. Very similar performances are achieved when the data are encoded in a normalized fashion (i.e. normalized scale encoding) or encoded in the binary form (i.e. 1-of-N encoding). Since it requires no additional process such as normalization or binary transformation, use of raw encoding would be preferable.

There are a number of studies in the literature that employ machine learning techniques to classify melanocytic lesions into common nevus, atypical nevus and melanoma using the dermoscopic measures of the lesions mentioned above. The findings demonstrate that artificial neural networks perform better than support vector machines, K-nearest neighbor classifiers and also decision tree classifiers $[10,11]$. A feed-forward neural network having eighteen neurons in the hidden layer is illustrated to offer $92.5 \%$ accuracy when fed by the dermoscopic measures encoded using 1-of-N encoding scheme [11]. On the other hand, a deep neural network with a Softmax activation function is reported to achieve $91.9 \%$ classification accuracy for the measures encoded using normalized scale encoding [10]. Meanwhile, a feed-forward neural network with fifteen neurons in the hidden layer is reported to offer $93.3 \%$ classification accuracy for the measures applied with raw encoding method [9]. This network is equipped with a linear activation function at the output layer. In the current study, we report an improved accuracy of $98.0 \%$ from a feed-forward neural network with seven neurons in the hidden layer for the measures applied with raw encoding method. This network houses a Softmax activation function at the output layer.

The Softmax activation function is habitually employed in the output layer of the neural networks aiming at multiclass classification [17]. Therefore, its use in the network developed during the current study makes the classification of the three different types of melanocytic lesions possible. On the other hand, the output of a Softmax activation function is a probability distribution. Accordingly, cross-entropy loss function was used during the training of the designed network. Due to the cooperative use of cross-entropy loss function and Softmax activation function, the network obtained carries out a nonlinear variant of multinomial logistic regression that leads to an improved classification performance with a small number of neurons in the hidden layer.
There are some limitations of the current study. The results are for the melanocytic lesions from the $\mathrm{PH} 2$ dataset that delivers histological diagnoses only for the lesions considered highly suspicious by dermatologists. The dataset offers several dermoscopic measures but measures for border characteristics of the lesion, diameter of the lesion or occurrence of atypical vascular pattern in the lesion, are not covered which may allow for further improvements in the classification performance. The neural networks developed are all trained using scaled conjugate gradient optimization and cross-entropy loss function. Other optimization algorithms such as Marquardt-Levenberg and other loss functions such as mean square may improve the classification performance further.

In conclusion, the classification of melanocytic lesions of the human skin, especially of melanomas, requires the utmost attention since melanoma is the most aggressive and life-threatening form of skin cancer. Early diagnosis is the key to decreasing the mortality rate. A feed-forward neural network fed by the lesion measures from dermoscopy can perform cancer detection while discriminating the non-cancerous types with quite high accuracy. However, for improved classification performance, the measures need to be encoded properly and then fed into the network. Practical use of such a network setup may facilitate lesion classification by minimizing the need for expertise and also by reducing the diagnostic variability among the dermatologists.

Peer-review: Externally peer-reviewed.

Conflict of Interest: The authors have no conflicts of interest to declare.

Financial Disclosure: The authors declared that this study has received no financial support.

\section{References}

1. P. Corrie, M. Hategan, K. Fife, C. Parkinson, "Management of melanoma", Br Med Bull, vol. 111, no. 1, pp. 149-162, 2014.

2. C. Cerchia, A. Lavecchia, "Small molecule drugs and targeted therapy for melanoma: Current strategies and future directions", Curr Med Chem, vol. 24, pp. 2312-2344, 2017.

3. V. Gray-Schopfer, C. Wellbrock, R. Marais, "Melanoma biology and new targeted therapy", Nature, vol. 445, no. 7130, pp. 851-857, 2007.

4. V. P. Silva, J. K. Ikino, M. M. Sens, D. H. Nunes, G. Di Giunta, "Dermoscopic features of thin melanomas: a comparative study of melanoma in situ and invasive melanomas smaller than or equal to 1mm", An Bras Dermatol, vol. 88, no. 5, pp. 712-717, 2013.

5. R. J. Friedman, D. S. Rigel, A. W. Kopf, "Early detection of malignant melanoma: the role of physician examination and self-examination of the skin", CA: Cancer J Clin, vol. 35, no. 3, pp. 130-151, 1985.

6. S. W. Menzies, C. Ingvar, K. A. Crotty, W. H. McCarthy, "Frequency and morphologic characteristics of invasive melanomas lacking specific surface microscopic features", Arch Dermatol, vol.132, no. 10, pp. 1178-1182, 1996.

7. G. Argenziano, G. Fabbrocini, P. Carli, V. De Giorgi, E. Sammarco, M. Delfino, "Epiluminescence microscopy for the diagnosis of 
doubtful melanocytic skin lesions: comparison of the $A B C D$ rule of dermatoscopy and a new 7-point checklist based on pattern analysis", Arch Dermatol, vol. 134, pp. 1563-1570, 1998.

8. C. Carrera, M. A. Marchetti S. W. Dusza, G. Argenziano, R. P. Braun, A. C. Halpern, N. Jaimes, H. J. Kittler, J. Malvehy, S. W. Menzies, G. Pellacani, S. Puig, H. S. Rabinovitz, A. Scope, H. P. Soyer, W. Stolz, R. Hofmann-Wellenhof, I. Zalaudek, A. A. Marghoob, "Validity and reliability of dermoscopic criteria used to differentiate nevi from melanoma: A web-based international dermoscopy society study", JAMA Dermatol, vol. 152, no. 7, pp 798-806, 2016.

9. U. Fidan, I. Sari, R. K. Kumrular, "Classification of skin lesions using ANN", Medical Technologies National Congress (TIPTEKNO), 2016, pp. 1-4.

10. A. Bastürk, M. E. Yuksel, H. Badem, A. Calışkan, "Deep neural network based diagnosis system for melanoma skin cancer", Signal Processing and Communications Applications Conference (SIU), 2017, pp. 1-4.

11. I. A. Özkan, M. Koklu, "Skin Lesion Classification using Machine Learning Algorithms", International Journal of Intelligent Systems and Applications in Engineering, vol. 5, no. 4, pp 285-289, 2017.
12. T. Mendonça, P. M. Ferreira, J. S. Marques, A. R. Marcal, J. Rozeira, "PH 2-A dermoscopic image database for research and benchmarking", Engineering in Medicine and Biology Society (EMBC), 35th Annual International Conference of the IEEE, 2013, pp. 54375440.

13. T. T. Wong, N. Y. Yang, "Dependency analysis of accuracy estimates in k-fold cross validation", IEEE Transactions on Knowledge and Data Engineering, vol. 29, no. 11, 2017.

14. M. F Moller, "A scaled conjugate gradient algorithm for fast supervised learning", Neural Networks, vol. 6, no. 4, pp. 525-533, 1993.

15. P. T. de Boer, D. P. Kroese, S. Mannor R. Y. Rubinstein, "A tutorial on the cross-entropy method", Annals of Operations Research, vol. 134, no. 1, pp. 19-67, 2005.

16. T. Kautz, B. M. Eskofier, C. F. Pasluosta, "Generic performance measure for multiclass-classifiers", Pattern Recognition, vol. 68, pp. 111125, 2017.

17. M. Nabian, "A comparative study on machine learning classification models for activity recognition", J Inform Tech Softw Eng, vol. 7, no. 4, p. 209, 2017. degrees from Bogazici University in Biomedical Engineering in 2001 and 2007, respectively. He led efforts in developing computational methods for comparing breast images at the Institute of Cancer Research and the Royal Marsden Hospital, UK. He is currently the vice chairman of the Biomedical Engineering Department at Yeditepe University. His current interests include neural networks as applied to medicine. 